\title{
Airborne Integrated Navigation System Based on SINS/GPS/TAN/EOAN
}

\author{
Junjun Tang $\mathbb{D}^{1}$ and Peijuan Li $\mathbb{D}^{2}$ \\ ${ }^{1}$ Stated-owned Wuhu Machinery Factory, Wuhu 241007, China \\ ${ }^{2}$ Institution School of Innovation \& Entrepreneurship, Nanjing Institute of Technology, Nanjing 211167, China \\ Correspondence should be addressed to Peijuan Li; lpj4444@126.com
}

Received 22 August 2020; Accepted 24 October 2020; Published 12 November 2020

Academic Editor: Guo Wei

Copyright ( $) 2020$ Junjun Tang and Peijuan Li. This is an open access article distributed under the Creative Commons Attribution License, which permits unrestricted use, distribution, and reproduction in any medium, provided the original work is properly cited.

\begin{abstract}
Considering the drawbacks that GPS signal is susceptible to obstacles and TAN becomes useless in some area when without any terrain data or with a featureless terrain field, to realize long-distance and high-precision navigation, a navigation system based on SINS/GPS/TAN/EOAN is presented. When GPS signal is available, GPS is used to correct errors of SINS; when GPS is unavailable, a terrain selection method based on the entropy weighted gray relational decision-making method is use to distinguish terrain into matchable areas and unmatchable areas; then, for the matchable areas, TAN is used to correct errors of SINS, for the unmatchable areas, EOAN is used to correct errors of SINS. The principles of SINS, GPS, TAN, and EOAN are analyzed, the mathematic models of SINS/GPS, SINS/TAN, and SINS/EOAN are constructed, and finally the federated Kalman filter is used to fuse navigation information. Simulation results show that the trajectory of SINS/GPS/TAN/EOAN is close to the ideal one in both matchable area or unmatchable area and whose navigation errors are obviously reduced, which is important for the realization of long-time and high-precision positioning.
\end{abstract}

\section{Introduction}

The Strapdown Inertial Navigation System (SINS) is an autonomous navigation system, which neither relies on any external information nor radiates energy to the outside world [1]. Besides, it has the advantages of high precision, good concealment, no susceptibility to interference, and so on. However, due to its navigation characteristics, the error of SINS inevitably grows with time. To realize long-distance and high-precision navigation, several aided navigation systems are used to correct its errors.

Global Positioning System (GPS) is a satellite-based radio navigation system that can provide accurate velocity and position information [2,3]. It has advantages of high precision, short observation time, and so on. However, it is susceptibility to interference, the signals might be blocked and attenuated, and when GPS information is unavailable, the error of SINS is unable to be corrected.
Terrain aided navigation system is an ideal auxiliary navigation method, which is based on terrain elevation characteristics $[4,5]$. The error of TAN is not accumulated while it has advantages of autonomy, concealment, continuity, and so on. However, it cannot achieve good effect in the areas where the terrain is flat or is of less terrain features. So, to make full use of TAN, it is necessary to distinguish terrain into matchable areas and unmatchable areas by measuring terrain feature parameters [6-9]. A novel selection criteria based on entropy weighted gray relational decision-making is used to divide terrain into matchable areas and unmatchable areas, in which the influences of terrain parameters such as standard deviation, roughness, entropy, and correlation coefficient are comprehensively considered.

Electro-optical Aided Navigation (EOAN) System is based on image matching technology that can provide position information [10]. It has advantages of high precision and strong anti-interference ability. For the area where 
there is less of terrain information, EOAN can be used to correct errors of SINS [11].

Integration navigation technology [12-15] has become one of the key issues because of its capability of overcoming the drawbacks of single navigation method as above $[16,17]$. And it is necessary to research multisensor fusion technologies [18]. In the multisensor information fusion system, distributed filters have attracted more and more attention of researchers $[19,20]$. The federated Kalman filter, which is one kind of the distributed filtering technique, is proposed by Carlson, and it has been widely used in multi-information fusion because of its flexibility and fault tolerance [15, 21-24].

The ideal of the integrated navigation system is shown as Figure 1. For the areas where GPS is available, GPS is used to correct errors of SINS; for the areas where GPS is unavailable but is of full terrain information, TAN is used to correct errors of SINS; and for the areas where GPS is unavailable and is of less terrain information, EOAN is used to correct errors of SINS.

The paper is organized as follows. In Section 2, terrain parameters are described and the selection method is introduced. In Section 3, the principles of SINS, GPS, TAN, and EOAN are analyzed and the mathematic models of SINS/GPS, SINS/TAN, and SINS/EOAN are constructed. In Section 4, the federated Kalman filter is proposed. In Section 5 , several tests are conducted to evaluate the performance of SINS/GPS/TAN/EOAN with FKF. And conclusions are made in Section 6.

\section{Terrain Selection Method}

2.1. Terrain Parameters. The terrain data is generally stored in the form of grid matrix. Assume that

(A) The exemplified terrain has $m \times n$ grids

(B) The coordinates of the grid point is $(i, j)$

(C) The height of the grid points is $h(i, j)$

Terrain parameters such as standard deviation, roughness, entropy, and correlation coefficient can be defined as follows:

2.1.1. Standard Deviation. The terrain standard deviation reflects the range of terrain elevation deviating from the average value. It is a macroparameter reflecting the severity of terrain overall fluctuation. And it can be defined as follows:

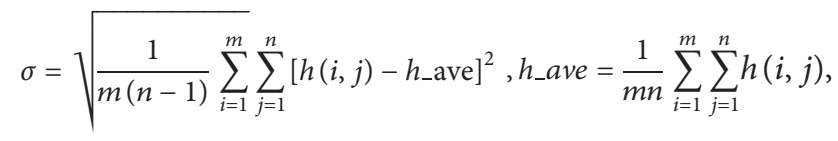

where $h_{-}$ave means the average value of terrain elevation.

2.1.2. Correlation Coefficient. Terrain correlation coefficient is a characteristic parameter used to reflect terrain correlation, and it can be defined as follows:

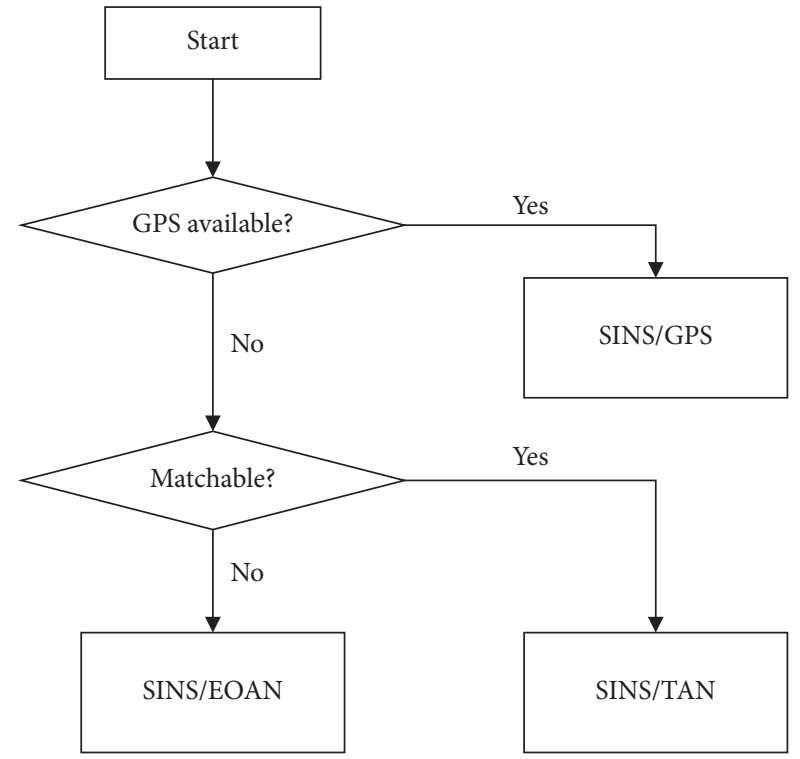

Figure 1: Airborne integrated navigation system.

$$
\begin{aligned}
R & =\frac{1}{2}\left(R_{\text {long }}+R_{\text {lati }}\right), \\
R_{\text {long }} & =\frac{1}{n(m-1) \sigma^{2}} \sum_{i=1}^{m-1} \sum_{j=1}^{n}\left[h(i, j)-h_{-} \text {ave }\right] \bullet\left[h(i+1, j)-h_{-} \text {ave }\right], \\
R_{\text {lati }} & =\frac{1}{n(m-1) \sigma^{2}} \sum_{i=1}^{m} \sum_{j=1}^{n-1}\left[h(i, j)-h_{-} \text {ave }\right] \bullet\left[h(i, j+1)-h_{-} \text {ave }\right],
\end{aligned}
$$

where $R_{\text {long }}$ means correlation coefficient of longitude direction, which reflects the correlation degree in latitude direction of terrain profile in longitude direction, and $R_{\text {lati }}$ means correlation coefficient of latitude, which reflects the correlation degree in longitude direction of terrain profile in latitude direction.

2.1.3. Roughness. Terrain roughness reflects the smoothness of terrain and is a characteristic parameter used to describe the local undulation, and it can be defined as follows:

$$
\begin{aligned}
r & =\frac{1}{2}\left(r_{\text {long }}+r_{\text {lati }}\right), \\
r_{\text {long }} & =\frac{1}{n(m-1)} \sum_{i=1}^{m-1} \sum_{j=1}^{n}|h(i, j)-h(i+1, j)|, \\
r_{\text {lati }} & =\frac{1}{m(n-1)} \sum_{i=1}^{m} \sum_{j=1}^{n-1}|h(i, j)-h(i, j+1)|,
\end{aligned}
$$

where $r_{\text {long }}$ represents the roughness of longitude and $r_{\text {lati }}$ represents the roughness of latitude.

2.1.4. Terrain Height Entropy. Terrain height entropy is a characteristic parameter reflecting the amount of information. The more obvious the terrain height changes, the 
smaller the height entropy. The height entropy can be described as follows:

$$
H_{f}=-\sum_{i=1}^{m} \sum_{j=1}^{n} p(i, j) \log p(i, j), p(i, j)=\frac{h(i, j)}{\sum_{i=1}^{m} \sum_{j=1}^{n} h(i, j)},
$$

where $p(i, j)$ means the normalized height value.

\subsection{Entropy Weighted Gray Relational Decision-Making.} Different parameters have different effects on the abundance of terrain information. Under certain conditions, the smaller the height entropy and the correlation coefficient are, the more abundant the terrain is and the greater the adaptability is. Conversely, the bigger the standard deviation and the roughness are, the more abundant the terrain is and the greater the adaptability is. Therefore, it is necessary to take all parameters into consideration and adopt a method which is able to fuse multifeatures. Assume that $T=\left(\sigma, r, H_{f}, R\right)$ is a feature vector after fused, according to which the adaptability of the terrain can be researched and a more comprehensive selection method is obtained.

2.2.1. Construction of Decision Matrix. Assume that the number of terrain candidate areas is $m$, which are $X_{1}, X_{2}, \ldots, X_{m}$, and the number of feature parameters in every candidate area is $n$, which are $G_{1}, G_{2}, \ldots G_{n}$. The property of candidate area in parameter is $x_{i j}$, and the decision matrix can be obtained:

$$
X=\left[\begin{array}{cccc}
x_{11} & x_{12} & \ldots & x_{1 n} \\
x_{21} & x_{22} & \ldots & x_{2 n} \\
\vdots & \vdots & \ddots & \vdots \\
x_{m 1} & x_{m 2} & \ldots & x_{m n}
\end{array}\right] .
$$

2.2.2. Normalized Matrix. Regarding correlation coefficient and height entropy as cost indicators and standard deviation and roughness as benefit indictors, the decision matrix $Y=$ $\left(y_{i j}\right)_{m \times n}$ can be normalized as follows:

$$
\left\{\begin{array}{l}
y_{i j}=\frac{x_{i j}-\min _{i} x_{i j}}{\max _{i} x_{i j}-\min _{i} x_{i j}} \text { (benefit indictors), } \\
y_{i j}=\frac{\max _{i} x_{i j}-x_{i j}}{\max _{i} x_{i j}-\min _{i} x_{i j}} \text { (cost indictors). }
\end{array}\right.
$$

2.2.3. Grey Relational Matrix. Assume that the optimal solution of indictors is $y_{j}^{*}, j=1,2, \ldots, n$, the reference sequence consisting of $y_{j}^{*}$, and can be presented as $y_{0}(j)=\left\{y_{j}^{*} \mid j=1,2, \ldots, n\right\}$. Making $y_{i}(j)=\left\{y_{i j} \mid j=1,2\right.$ $, \ldots, n\},(i=1,2, \ldots, m)$ to be comparison sequence, the correlation coefficient of $y_{i}$ and $y_{0}$ in indictor can be calculated as follows:

$$
\xi_{i}(j)=\frac{\Delta_{\min }+\rho \Delta_{\max }}{\Delta_{i 0}(j)+\rho \Delta_{\max }},
$$

where $\Delta_{\min }=\min \min \left|y_{0}(j)-y_{i}(j)\right|$ and $\Delta_{\max }=\max _{i}$ $\max \left|y_{0}(j)-y_{i}(j)\right|$, ${ }^{j}$ respectively, means the minimum value and the maximum value of absolute differences of comparison sequence. $\Delta_{i 0}(j)=\left|y_{0}(j)-y_{i}(j)\right|$ represents the absolute differences of comparison sequence and $\rho$ is the resolution coefficient, where $0<\rho<1$. Assume that $\rho=0.5$.

The gray relational matrix can be as follows:

$$
\xi=\left[\begin{array}{cccc}
\xi_{1}(1) & \xi_{1}(2) & \ldots & \xi_{1}(n) \\
\xi_{2}(1) & \xi_{2}(2) & \ldots & \xi_{2}(n) \\
\vdots & \vdots & \ddots & \vdots \\
\xi_{m}(1) & \xi_{m}(2) & \ldots & \xi_{m}(n)
\end{array}\right] .
$$

\subsubsection{Weight Calculation of Entropy}

(A) The calculation of entropy requires logarithm, to avoid meaningless data, the data in the normalized matrix can be translated by adding 1 , and the contribution of terrain $i$ to parameter $j$ can be calculated as follows:

$$
f_{i j}=\frac{y_{i j}+1}{\sum_{i=1}^{m}\left(y_{i j}+1\right)} \text {. }
$$

(B) The entropy $E_{j}$ can be used to represent the total contribution of all terrains to indictor $j$ :

$$
E_{j}=-k \sum_{i=1}^{m} f_{i j} \ln f_{i j}, \quad(j=1,2, \ldots, n),
$$

where $k>0$, and assume that $k=1 / \ln m$, $E_{j} \in[0,1]$. For conditions that the contributions of each terrain to indictor $j$ is consistent, $E_{j}$ tends to be 1 , and the indictor is useless in the decision-making, and the weight value can be 0 . Reversely, for conditions that the contributions of each terrain to indictor $j$ are quietly different, the indictor is more useful to decision-making, and the weight value should be lager.

(C) Define $g_{j}$ as the difference coefficient of contribution of terrains to indictor $j$, and the indictor weight can be calculated as follows:

$$
\begin{gathered}
g_{j}=1-E_{j}, \quad(j=1,2, \ldots, n), \\
w_{j}=\frac{g_{j}}{\sum_{j=1}^{n} g_{j}}, \quad(j=1,2, \ldots, n) .
\end{gathered}
$$

\subsubsection{Comprehensive Evaluation Value of Gray Correlation} Degree. The comprehensive evaluation value of gray correlation degree of terrain $X_{i}$ is as follows: 


$$
D_{i}=\sum_{j=1}^{n} \xi_{i}(j) w_{j} .
$$

The comprehensive evaluation values can be sorted; the bigger the evaluation value is, the higher the adaptability of the terrain is.

\section{Mathematical Model}

3.1. Error Model of SINS. Here, we define the navigation coordinates frames as an east-north-vertical geography coordinates frames. The position errors, velocity errors, attitude angle errors, gyro drift, and accelerometer bias are formed as the state vector. The state vector is determined by

$$
X_{\mathrm{SINS}}=\left[\delta L \delta \lambda \delta V_{E} \delta V_{N} \Phi_{E} \Phi_{N} \Phi_{U} \nabla_{b x} \nabla_{b y} \varepsilon_{b x} \varepsilon_{b y} \varepsilon_{b z}\right]^{T}
$$

where $\delta L$ and $\delta \lambda$ represent the position errors, $\delta V_{E}$ and $\delta V_{N}$ are east and north velocity errors, $\varphi_{E}, \varphi_{N}$, and $\varphi_{U}$ are misalignment angles of pitch, roll, and yaw, $\nabla_{b x}$ and $\nabla_{b y}$ represent accelerometer bias, and $\varepsilon_{b x}, \varepsilon_{b y}$, and $\varepsilon_{b z}$ are gyro drifts along $x, y$, and $z$ axes.

The system state equation can be constructed as

$$
\dot{X}_{\text {SINS }}=F_{\text {SINS }} X_{\text {SINS }}+W_{\text {SINS }} \text {, }
$$

where

$$
\begin{aligned}
& W_{\mathrm{SINS}}=\left[\begin{array}{llllllllllll}
\omega_{\delta_{L}} & \omega_{\delta_{\lambda}} & \omega_{V_{E}} & \omega_{V_{N}} & \omega_{\phi_{E}} & \omega_{\phi_{N}} & \omega_{\phi_{U}} & 0 & 0 & 0 & 0 & 0
\end{array}\right], \\
& F_{21}=\frac{V_{E}}{R} \sec ^{2} L \sin L \\
& F_{31}=\left(2 \omega_{i e} \cos L+\frac{V_{E}}{R} \sec ^{2} L\right) V_{N} \text {, } \\
& F_{41}=-\left(2 \omega_{i e} \cos L+\frac{V_{E}}{R} \sec ^{2} L\right) V_{E}, \\
& F_{34}=2 \omega_{i e} \sin L+\frac{V_{E}}{R} \tan L \\
& F_{43}=-2\left(\omega_{i e} \sin L+\frac{V_{E}}{R} \tan L\right), \\
& F_{57}=-F_{75}=-\left(\omega_{i e} \cos L+\frac{V_{E}}{R}\right) \text {, } \\
& F_{56}=-F_{65}=\omega_{i e} \sin L+\frac{V_{E}}{R} \tan L, \\
& F_{61}=-\omega_{i e} \sin L, \\
& F_{71}=\omega_{i e} \cos L+\frac{V_{E}}{R} \sec ^{2} L .
\end{aligned}
$$

$C_{i j}$ is element of $C_{n}^{b}$, which is the direction cosine matrix

from $n$-frame to $b$-frame: 


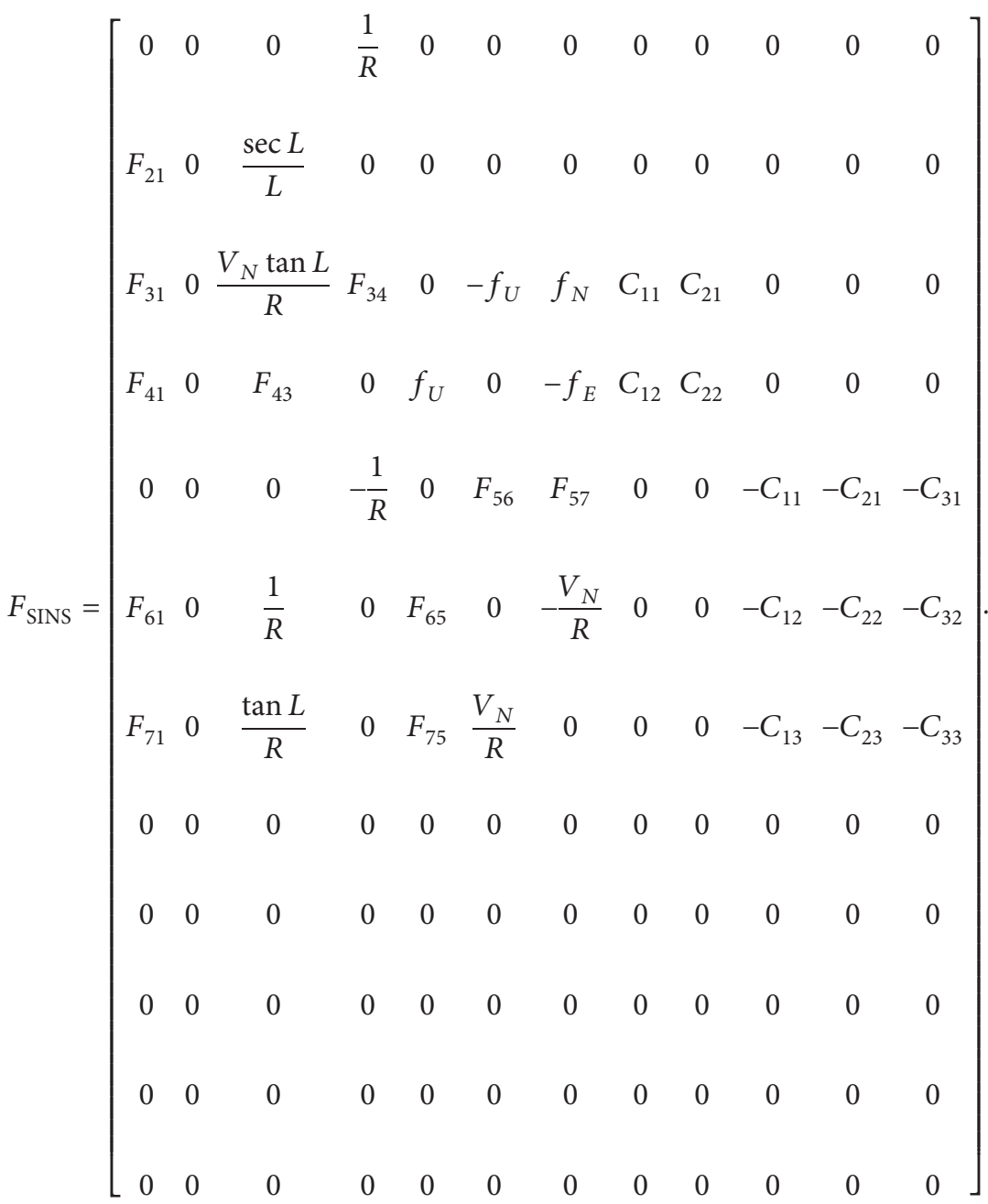

3.2. Mathematical Model of SINS/GPS. In the areas where GPS is available, GPS is used to correct errors of SINS. Take the difference between the position and velocity information of the INS and the GPS as the observation measurement and establish the system measurement equation as follows:

$$
Z_{k_{-} \mathrm{GPS}}=\left[\begin{array}{c}
L_{\mathrm{SINS}}-L_{\mathrm{GPS}} \\
\lambda_{\mathrm{SINS}}-\lambda_{\mathrm{GPS}} \\
V_{E_{-} \mathrm{SINS}}-V_{E_{-} \mathrm{GPS}} \\
V_{N_{-} \mathrm{SINS}}-V_{N_{-} \mathrm{GPS}}
\end{array}\right]=H_{k_{-} \mathrm{GPS}}\left[\begin{array}{c}
X_{\mathrm{SINS}} \\
X_{\mathrm{GPS}}
\end{array}\right]+V_{k_{-} \mathrm{GPS}},
$$

where $L_{\text {SINS }}, \lambda_{\text {SINS }}, V_{E_{-} \text {SINS }}$, and $V_{N_{-} \text {SINS }}$ are position and velocity information of SINS, $L_{\mathrm{GPS}}, \lambda_{\mathrm{GPS}}, V_{E_{-} \mathrm{GPS}}$, and $V_{N_{-} \mathrm{GPS}}$ are position and velocity information of GPS, $V_{k_{-} G P S}$ is the measurement noise matrix, and $H_{k_{-} G P S}$ is the measurement matrix, and the measurement matrix can be expressed as

$$
H_{k_{-} G P S}=\left[\begin{array}{cccccccccccccccccc}
1 & 0 & 0 & 0 & 0 & 0 & 0 & 0 & 0 & 0 & 0 & 0 & 0 & 0 & -1 & 0 & 0 & 0 \\
0 & 1 & 0 & 0 & 0 & 0 & 0 & 0 & 0 & 0 & 0 & 0 & 0 & 0 & 0 & -1 & 0 & 0 \\
0 & 0 & 1 & 0 & 0 & 0 & 0 & 0 & 0 & 0 & 0 & 0 & 0 & 0 & 0 & 0 & -1 & 0 \\
0 & 0 & 0 & 1 & 0 & 0 & 0 & 0 & 0 & 0 & 0 & 0 & 0 & 0 & 0 & 0 & 0 & -1
\end{array}\right] .
$$

3.3. Mathematical Model of SINS/TAN. In the areas where GPS is unavailable but is of enough terrain information, TAN is used to correct errors of SINS. The aircraft is assumed to be equipped with a radio altimeter to collect real-time height data during the navigation. According to the height map stored in advance, the matching algorithm is able to find the best nearest point, whose longitude and latitude are the output of TAN. Take the difference between the position information of the INS and the TAN as the observation measurement and establish the system measurement equation as follows:

$$
Z_{k_{-} \mathrm{TAN}}=\left[\begin{array}{c}
L_{\mathrm{SINS}}-L_{\mathrm{TAN}} \\
\lambda_{\mathrm{SINS}}-\lambda_{\mathrm{TAN}}
\end{array}\right]=H_{k_{-} \mathrm{TAN}}\left[\begin{array}{c}
X_{\mathrm{SINS}} \\
X_{\mathrm{TAN}}
\end{array}\right]+V_{k_{-} \mathrm{TAN}}
$$

where $L_{\text {TAN }}$ and $\lambda_{\text {TAN }}$ are position information outputted by TAN. And $V_{k_{-} \text {TAN }}$ is the measurement noise matrix and $H_{k_{-} \text {TAN }}$ is the measurement matrix, which can be expressed as

$$
H_{k_{-} \text {TAN }}=\left[\begin{array}{cccccccccccccc}
1 & 0 & 0 & 0 & 0 & 0 & 0 & 0 & 0 & 0 & 0 & 0 & -1 & 0 \\
0 & 1 & 0 & 0 & 0 & 0 & 0 & 0 & 0 & 0 & 0 & 0 & 0 & -1
\end{array}\right] .
$$


3.4. Mathematical Model of SINS/EOAN. In the areas where GPS is unavailable and is of less terrain information, EOAN is used to correct errors of SINS [11]. The aircraft is assumed to be equipped with a video camera to collect real-time image data during the navigation. Compared with the image database stored in advance and according to the hypothesis that the gray value of each point in the above image is equal, the distance between the target and aircraft can be calculated, and the real-time position of the aircraft can be converted. Take the difference between the position information of the INS and the EOAN as the observation measurement and establish the system measurement equation as follows:

$$
Z_{k_{-} \mathrm{EOAN}}=\left[\begin{array}{c}
L_{\mathrm{SINS}}-L_{\mathrm{EOAN}} \\
\lambda_{\mathrm{SINS}}-\lambda_{\mathrm{EOAN}}
\end{array}\right]=H_{k_{-} \mathrm{EOAN}}\left[\begin{array}{c}
X_{\mathrm{SINS}} \\
X_{\mathrm{EOAN}}
\end{array}\right]+V_{k_{-} \mathrm{EOAN}}
$$

where $L_{\mathrm{EOAN}}$ and $\lambda_{\mathrm{EOAN}}$ are position information obtained by EOAN, $V_{k_{-} \text {EOAN }}$ is the measurement noise matrix, and $H_{k_{-} \text {EOAN }}$ is the measurement matrix, which can be expressed as

$$
H_{k_{-} \text {EOAN }}=\left[\begin{array}{cccccccccccccc}
1 & 0 & 0 & 0 & 0 & 0 & 0 & 0 & 0 & 0 & 0 & 0 & -1 & 0 \\
0 & 1 & 0 & 0 & 0 & 0 & 0 & 0 & 0 & 0 & 0 & 0 & 0 & -1
\end{array}\right] .
$$

\section{Federated Kalman Filter}

4.1. System Scheme. The system scheme is shown in Figure 2. SINS is the public system, which provides position, velocity, and attitude angle information. The first subfilter is composed of SINS and GPS, the second subfilter is composed of SINS and TAN, and the third subfilter is composed of SINS and EOAN. All the above three subfilters are Kalman filters. The federal filter is composed of the three subfilters and a main filter, where the subfilter can independently update the time and measurement, and the result will be sent to the main filter. And the main filter will feed back to the subfilters after data fusion. It is the initial value of the next cycle. It is important that the local estimations are not related to each other, so the system has good fault tolerance and can meet the requirement of long-distance and high-precision navigation.

4.2. The Traditional Kalman Filter. It is assumed that the system equation and measurement equation of the linear discrete system can be written as follows:

$$
\begin{aligned}
& X_{i}(k)=\Phi_{i}\left(\frac{k}{k-1}\right) X_{i}(k-1)+W_{i}(k), \\
& Z_{i}(k)=H_{i}(k) X_{i}(k)+V_{i}(k),
\end{aligned}
$$

where $X_{i}(k)$ is the estimated state of subfilter $i, Z_{i}(k)$ is the measurement of subfilter $i, \Phi_{i}(k / k-1)$ is the transfer matrix from $t_{\mathrm{k}-1}$ to $\mathrm{t}_{\mathrm{k}}, W_{i}(k)$ is noise sequence of system incentive, $H_{i}(k)$ is the measurement matrix, $V_{i}(k)$ is noise sequence of measurement, and $E\left[V_{i}(k) V_{i}^{T}(k)\right]=R_{i}(k)$.

So, the subfilter equation is shown as follows.

(1) Time update is

$$
\left\{\begin{array}{l}
\widehat{X}_{i}\left(\frac{k}{k-1}\right)=\Phi_{i}\left(\frac{k}{k-1}\right) \widehat{X}_{i}(k-1), \\
P_{i}\left(\frac{k}{k-1}\right)=\Phi_{i}\left(\frac{k}{k-1}\right) P_{i}(k-1) \Phi_{i}^{T}\left(\frac{k}{k-1}\right)+\Gamma_{i}(k-1) Q_{i}(k-1) \Gamma_{i}^{T}(k-1) .
\end{array}\right.
$$

(2) Measurement update is

$$
\left\{\begin{array}{l}
K_{i}(k)=P_{i}\left(\frac{k}{k-1}\right) H_{i}^{T}(k)\left(H_{i}^{T}(k) P_{i}\left(\frac{k}{k-1}\right) H_{i}^{T}(k)+R_{i}(k)\right)^{-1} \\
P_{i}\left(\frac{k}{k}\right)=\left(I-K_{i}(k) H_{i}(k)\right) P_{i}\left(\frac{k}{k}-1\right)\left[I-K_{i}(k) H_{i}(k)\right]^{T}+K_{i}(k) R_{i}(k) K_{i}^{T}(k), \\
\widehat{X}_{i}\left(\frac{k}{k}\right)=\widehat{X}_{i}\left(\frac{k}{k-1}\right)+K_{i}(k)\left[Z_{i}(k)-H_{i}\left(\frac{k}{k}\right) \widehat{X}_{i}\left(\frac{k}{k-1}\right)\right]
\end{array}\right.
$$




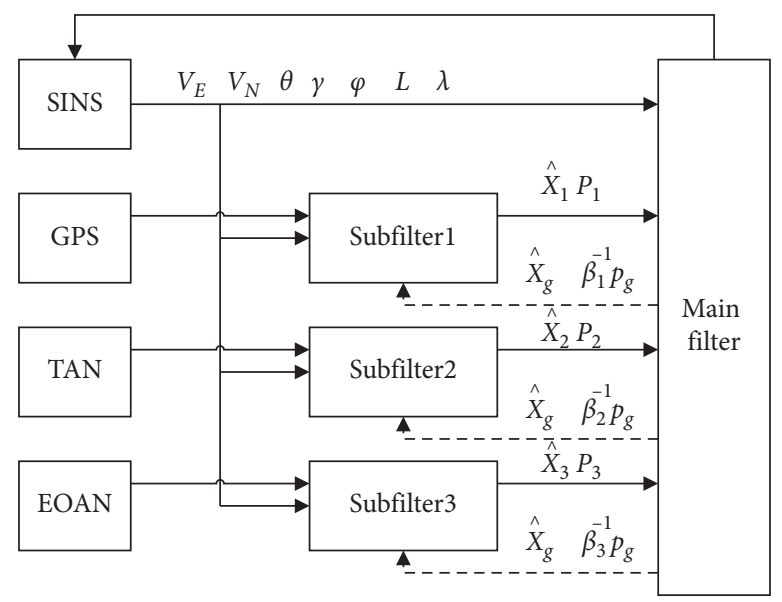

Figure 2: Design structure of the filter.

4.3. The Federal Filter. The fusion algorithm of the federal filter [18] is as follows:

$$
\left\{\begin{array}{l}
P_{g}=\left(\sum_{i=1}^{3} P_{i}^{-1}\left(\frac{k}{k}\right)\right)^{-1}, \\
\hat{X}_{g}=P_{g}\left(\sum_{i=1}^{3} P_{i}^{-1}\left(\frac{k}{k}\right) \hat{X}_{i}\left(\frac{k}{k}\right)\right),
\end{array}\right.
$$

where $\widehat{X}_{q}$ is global state estimation after fusion, $\widehat{X}_{i}(k / k)$ is the local optimum estimation of subfilter, $P_{g}$ is the variance matrix of error, and $P_{i}(k / k)$ is the local optimum estimation.

4.4. Information Distribution. The principle of information distribution in the federated Kalman filter is

$$
\begin{aligned}
& \left\{\begin{array}{l}
P_{i}^{-1}(k)=\beta_{i} P_{g}^{-1}(k) \\
\widehat{X}_{i}(k)=\widehat{X}_{g}(k) \\
Q_{i}^{-1}(k)=\beta_{i} Q_{g}^{-1}(k)
\end{array} \quad(i=1,2,3),\right. \\
& \sum_{i=1}^{3} \beta_{i}+\beta_{m}=1,
\end{aligned}
$$

where $\beta_{i}$ represents the information distribution factor of subfilter and $\beta_{m}$ represents the information distribution factor of main filter, choosing $\beta_{m}=0$.

The estimation accuracy of the Federal filter is equivalent to that of the Kalman filter; however, the Federal filter has a small amount of calculation and is of strong fault tolerance. And distribution coefficient is the key to the performance of the federal filter. Considering that the estimation accuracy of each subfilter is decided by the error covariance matrix, the distribution coefficient can be determined be the eigenvalue of the error covariance matrix; thus,

$$
\beta_{i}(k)=\frac{\operatorname{tr} P_{i}(k-1)^{-1}}{\sum_{i=1}^{3} \operatorname{tr} P_{i}(k-1)^{-1}} .
$$

\section{Simulation Experiment}

The simulation terrain can be divided into matchable areas and unmatchable areas using the terrain selection method based on entropy weighted gray relational decision-making, and the map of divided terrain is shown as Figure 3.

The simulation conditions and parameters can be set as Table 1.

In the matchable area, the ideal trajectory starts from $(31.0325 \mathrm{~N}, 118.0300 \mathrm{E})$ and ends at $(31.0435 \mathrm{~N}, 118.0390 \mathrm{E})$. And the simulated navigation trajectories of SINS, SINS/ TAN, and the proposed method are shown in Figure 4.

And errors of trajectories in the matchable area are calculated as Table 2 .

In the unmatchable area, the ideal trajectory starts from $(31.0724 \mathrm{~N}, 118.0750 \mathrm{E})$ and ends at $(31.0834 \mathrm{~N}, 118.0840 \mathrm{E})$. And the simulated navigation trajectories of SINS, SINS/ TAN, and the proposed method are shown in Figure 5.

And errors of trajectories in the unmatchable area are calculated as Table 3.

In Figures 3 and 4, the red line represents ideal trajectory, the blue line represents SINS trajectory, the green line represents SINS/TAN trajectory, and the orange line represents trajectory of the proposed method. Obviously, the presented navigation method can obtain a trajectory which is much closer to the ideal one.

It can be seen from Table 2 that SINS/TAN can effectively reduce the positioning error in the matchable area, 


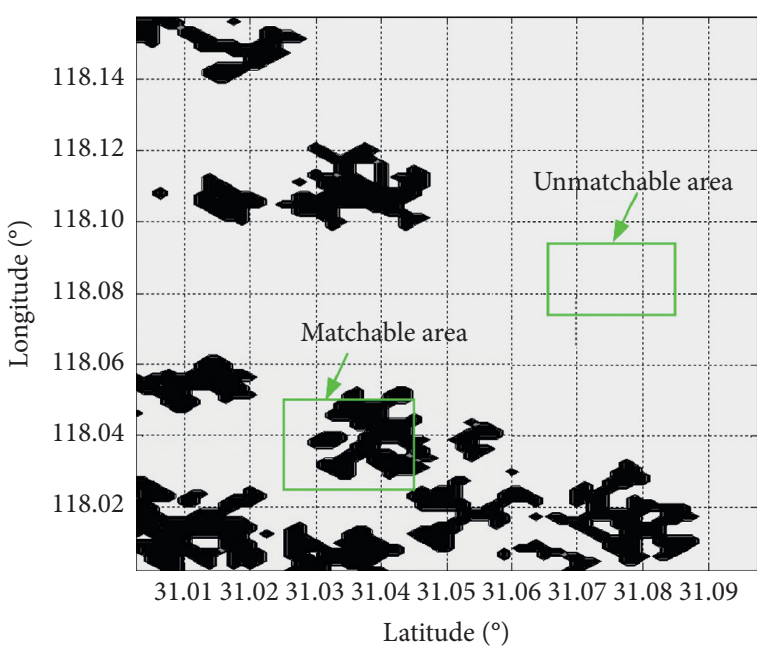

FIgURE 3: Map of divided terrain.

TABLE 1: Simulation conditions and parameters.

\begin{tabular}{lc}
\hline Terms & Conditions and parameters \\
\hline Latitude initial error of SINS (") & 5 \\
Longitude initial error of SINS (") & 5 \\
East velocity error (m/s) & 0.1 \\
North velocity error (m/s) & 0.1 \\
Accelerometer biases (ug) & 20 \\
Gyroscope constant ( $/ \mathrm{h})$ & 0.1 \\
Positioning error of TAN (m) & 50 \\
Positioning error of EOAN (m) & 50
\end{tabular}

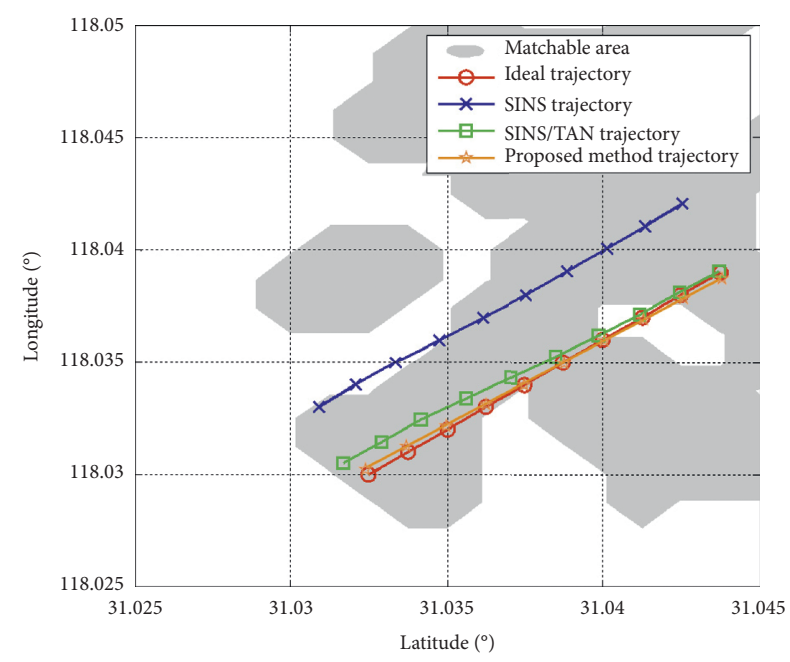

Figure 4: Navigation trajectory in the matchable area.

TABLE 2: Errors of trajectories in the matchable area.

\begin{tabular}{lcccc}
\hline \multirow{2}{*}{ Trajectory } & \multicolumn{2}{c}{ Errors of latitude } & \multicolumn{2}{c}{ Errors of longitude } \\
& Maximum & Average & Maximum & Average \\
\hline SINS & 186.5326 & 151.0221 & 344.6527 & 337.2541 \\
SINS/TAN & 95.7125 & 45.6324 & 57.8921 & 35.2196 \\
SINS/GPS/TAN/ & 88.3069 & 42.6781 & 55.6326 & 34.1945 \\
EOAN & & & & \\
\hline
\end{tabular}

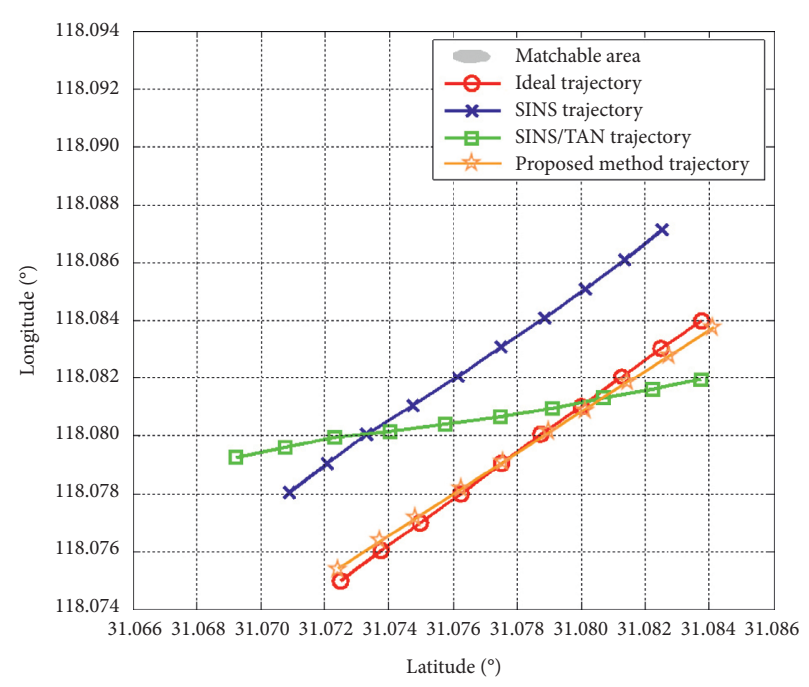

Figure 5: Navigation trajectory in the unmatchable area.

TABLE 3: Errors of trajectories in the unmatchable area.

\begin{tabular}{lcccc}
\hline \multirow{2}{*}{ Trajectory } & \multicolumn{2}{c}{ Errors of latitude } & \multicolumn{2}{c}{ Errors of longitude } \\
& Maximum & Average & Maximum & Average \\
\hline SINS & 186.5326 & 151.0221 & 344.6527 & 337.2541 \\
SINS/TAN & 350.1225 & 177.5213 & 465.2315 & 215.2196 \\
SINS/GPS/TAN/ & 90.1743 & 41.5698 & 59.3249 & 36.3312 \\
EOAN & & & & \\
\hline
\end{tabular}

while the positioning accuracy of SINS/GPS/TAN/EOAN is slightly higher than that of SINS/TAN.

From Table 3, it can be seen that SINS/TAN becomes useless in the unmatchable area, while SINS/GPS/TAN/ EOAN can still significantly improve the positioning accuracy.

\section{Conclusion}

The airborne integrated navigation system proposed in this paper is a combination of SINS, GPS, TAN, and OEAN. The federal Kalman filter is used to fuse the navigation information. Compared with the simulation results, it can be seen that there is a lager deviation between the trajectory of pure SINS and that of ideal one; the trajectory of TAN is close to the ideal one in only the matchable area, while the trajectory of SINS/GPS/TAN/EOAN is close to the ideal one in both matchable area or unmatchable area, and whose navigation errors are obviously reduced, which is important for the realization of long-time and high-precision positioning.

\section{Data Availability}

The data used to support the findings of this study are included within the article.

\section{Conflicts of Interest}

The authors declare that there are no conflicts of interest regarding the publication of this paper. 


\section{Acknowledgments}

The authors would like to thank the Scientific Research Foundation of Nanjing Institute of Technology, the introduction of talent project (Grant no. YKJ201531).

\section{References}

[1] X. S. Xu, J. J. Tang, T. Zhang et al., "Under-water navigation method based on terrain and environmental features,", Journal of Chinese Inertial Technology, vol. 23, no. 5, pp. 590-596, 2015, in Chinese.

[2] G. Hu, B. Gao, and Y. Zhong, "Unscented kalman filter with process noise covariance estimation for vehicular ins/gps integration system," Information Fusion, vol. 64, pp. 194-204, 2020.

[3] G. Hu, L. Ni, B. Gao, W. Wang, and Y. Zhong, "Model predictive based unscented kalman filter for hypersonic vehicle navigation with INS/GNSS integration," IEEE Access, vol. 8, pp. 4814-4823, 2020.

[4] J. J. Tang, W. Hu, X. S. Liu et al., "Unmanned aerial vehicle integrated navigation system based on SINS/TAN/ADS/ MCP," Journal of Chinese Inertial Technology, vol. 26, no. 1, pp. 33-38, 2018, inChinese.

[5] S. Gu, H. Zhang, J. Zhao, and P. Tang, "Marine geomagnetic navigation technology based on integration of TERCOM and ICCP," Geomatics and Information Science of Wuhan University, vol. 36, no. 10, pp. 1209-1212, 2011.

[6] A. Bar-Gill, P. Ben-Ezra, and I. Y. Bar-Itzhack, "Improvement of terrain-aided navigation via trajectory optimization," IEEE Transactions on Control Systems Technology, vol. 2, no. 4, pp. 336-342, 1994.

[7] X. S. Cao, J. F. Wu, S. B. Xu, L. H. Wang, and P. J. Li, "ICCP algorithm for underwater terrain matching navigation based on affine correction," Journal of Chinese Inertial Technology, vol. 22, no. 3, pp. 362-367, 2014.

[8] N. Bergman, L. Ljung, and F. Gustafsson, "Terrain navigation using Bayesian statistics," IEEE Control Systems Magazine, vol. 19, no. 3, pp. 33-40, 1999.

[9] C. Shen, Y. Zhang, X. Gao et al., "Seamless GPS/inertial navigation system based on self-learning square-root cubature kalman filter," IEEE Transactions on Industrial Electronics, vol. 17, 2020.

[10] Z. J. Zhu, J. X. Ren, and D. Li, "Inertial navigation assistance to electro-optical measurement and error analysis," Journal of Chinese Inertial Technology, vol. 14, no. 1, pp. 7-11, 2006, in Chinese.

[11] P. K. A. Menon, G. B. Chatterji, and B. Sridhar, "Electrooptical navigation for aircraft," IEEE Transactions on Aerospace and Electronic System, vol. 29, no. 2, pp. 825-832, 1993.

[12] S. A. Gu and A. J. Henley, "An integrated navigation system manager using federated Kalman filtering," in Proceedings of the IEEE National Aerospace and Electronics Conference (NAECON'91), pp. 422-426, Dayton. OH, USA, May 1991.

[13] M. Ushaq and F. J. Cheng, "A fault tolerant integrated navigation scheme realized through online tuning of weighting factors for federated Kalman filter," Applied Mechanics and Materials, vol. 446-447, pp. 1078-1085, 2014.

[14] X. Gao, W. Sun, Q. Li et al., "INS/Scene matching integrated navigation based on evidence reasoning," Journal of Chinese Inertial Technology, vol. 22, no. 5, pp. 624-628, 2014, in Chinese.

[15] U. K. Krogmann, "Failure management in spatio-temporal redundant, integrated navigation and flight control reference- systems," in Proceedings of the IEEE Position Location and Navigation Symposium, pp. 330-337, Atlanta, Georgia, March 1990.

[16] X. J. Deng, H. W. Liu, D. Xiao, and H. K. Li, "Key technologies of geomagnetic aided inertial navigation system," in Proceedings of the 2009IEEE Intelligent Vehicles Symposium, pp. 464-469, IEEE, Xi'an, China, June 2009.

[17] S. Gao, L. Xue, and Y. Zhong, "Random weighting method for estimation of error characteristics in SINS/GPS/SAR integrated navigation system," Aerospace Science and Technology, vol. 46, pp. 22-29, 2015, in Chinese.

[18] D. N. Jiang and T. M. Mekonnen, "INS/GPS/MNS Integrated navigation system with federated Kalman filtering," Journal of Advanced Materials Research, vol. 718-720, pp. 1207-1212, 2013.

[19] H. Miller and D. A. Hilts, "Fault tolerant integrated inertial navigation/global positioning systems for next generation spacecraft," in Proceedings of the IEEE/AIAA 10th Digital Avionics Systems Conference, pp. 207-212, Los Angeles, Calif, USA, October 1991.

[20] H. Deng, G. B. Liu, and H. Chen, "The application of federated kalman filtering in SINS/GPS/CNS intergrated navigation system," International Journal of Wireless and Microwave Technologies, vol. 2, no. 2, pp. 12-19, 2012.

[21] G. Hu, W. Wang, Y. Zhong, and C. Gu, "A new direct filtering approach to INS/GNSS integration," Aerospace Science and Technology, vol. 77, pp. 755-764, 2018.

[22] T. L. Xu, "Research on federated kalman filter for navigation system," in Proceeding of 2011 Third Pacific-Asia Conference on Circuits, Communications and System, pp. 4, Wuhan, China, July 2014.

[23] C. Shen, Y. Zhang, J. Tang, and J. Liu, "Dual-optimization for a MEMS-INS/GPS system during GPS outages based on the cubature Kalman filter and neural networks," Mechanical Systems and Signal Processing, vol. 133, Article ID 106222, 2019.

[24] X. Jin-Hua, X. Jing-Ning, T. Zhu et al., "Reduced-order EKF application in A marine INS/GPS navigation system," Acta Armamentrarii, vol. 27, no. 4, pp. 659-664, 2006. 